\title{
Precise detection of genomic imbalances at single- cell resolution reveals intra-patient heterogeneity in Hodgkin's lymphoma
}

\author{
Chiara Mangano ${ }^{1}$, Alberto Ferrarini', Claudio Forcato ${ }^{1}$, Marianna Garonzi ${ }^{1}$, Paola Tononi ${ }^{1}$, Rossana Lanzellotto ${ }^{1}$, \\ Andrea Raspadori ${ }^{1}$, Chiara Bolognesi ${ }^{1}$, Genny Buson ${ }^{1}$, Gianni Medoro ${ }^{1}$, Michael Hummel ${ }^{2}$, Francesca Fontana ${ }^{1}$ and \\ Nicolò Manaresi ${ }^{1}$
}

Dear Editor,

Classical Hodgkin lymphoma (cHL) is a B-cell-derived lymphoproliferative disorder ${ }^{1}$ characterized by the presence of morphologically characteristic pathognomonic malignant cells termed Hodgkin and Reed-Sternberg (HRS) cells. HRS cells are derived from germinal center B cells and represent only a small fraction (usually $<5 \%$ ) of all the cells in the tumor tissue, whereas the surrounding inflammatory milieu is rich in T cells, B cells, granulocytes, eosinophils, macrophages, and stromal cells ${ }^{2}$.

Despite the extensive inflammatory microenvironment, HRS cells are able to escape immune surveillance using several mechanisms, including overexpression of programmed cell death protein-1 (PD-1) ligands (PD-Ls), upregulated in a dose-dependent manner by copynumber alterations (CNAs) of chromosome 9p24.1, a locus that encodes PD-L1/PD-L2 as well as Janus kinase 2 (JAK2), which further enhances PD-L expression through the JAK2/STAT (signal transducer and activator of transcription) pathway. PD-L locus amplification has been associated with advanced stages of the disease and with a shorter progression-free survival ${ }^{3,4}$. Along with PD-L locus copy alterations, HRS cells display a general abnormal karyotype, including gains and losses extended evenly to whole chromosomes ${ }^{3}$. Genetic alterations of HRS cells are a valuable source of information to develop new treatments or predictive/prognostic biomarkers. However, rareness of HRS cells dispersed among the

Correspondence: Nicolò Manaresi (nmanaresi@siliconbiosystems.com)

${ }^{1}$ Menarini Silicon Biosystems S.p.A, Bologna, Italy

${ }^{2}$ Charité - Universitätsmedizin Berlin, Institut für Pathologie, Berlin, Germany

These authors contributed equally: Chiara Mangano, Alberto Ferrarini surrounding inflammatory milieu poses technical challenges to unravel malignant cells' genetic alterations.

To enable isolation and molecular characterization of HRSs without interference from nonmalignant cells, we set up an optimized workflow to isolate single HRSs starting from formalin-fixed paraffin-embedded (FFPE) tissue biopsies of cHL patients by disaggregation to a single-cell suspension and staining using membrane protein CD30 for HRS cell identification (for protocol details see Supplementary Methods and Supplementary Figs. 1-3).

To isolate single HRS cells, we leveraged on DEPArray $^{\mathrm{TM}}$, an image-based cell-sorting technology, previously successfully applied for single-cell isolation in liquid biopsy ${ }^{5,6}$ and for characterization of pools of cells from FFPE tissues ${ }^{7}$. In addition, we carried out single FFPE cell genomic profiling, based on Ampli1 ${ }^{\mathrm{TM}}$ WGA and Ampli $1^{\mathrm{TM}}$ LowPass, previously applied to single-cell liquid biopsy ${ }^{5,6}$. The proposed method shows as a proof of concept that it is possible to identify genome-wide CNAs at the singlecell level and obtain information about inter-tumor and intra-tumor heterogeneity.

The immunofluorescent pattern obtained for HRS cell identification is shown in Supplementary Fig. 4: CD30 signal is clearly localized in the cytoplasmic/membrane compartment and allows discriminating CD30positive HRS cells from CD30-negative cells.

DEPArray $^{\mathrm{TM}}$ digital sorting, a highly automated imagebased platform, allowed us to identify and recover whole pure HRS cells combining multiple marker expression and localization and cell morphology. Indeed, HRS cells have generally a polyploid genome/multinuclear appearance, and consequently, a higher DNA content compared with leukocytes. Thus, DAPI (4',6-diamidino-2-phenylindole) 


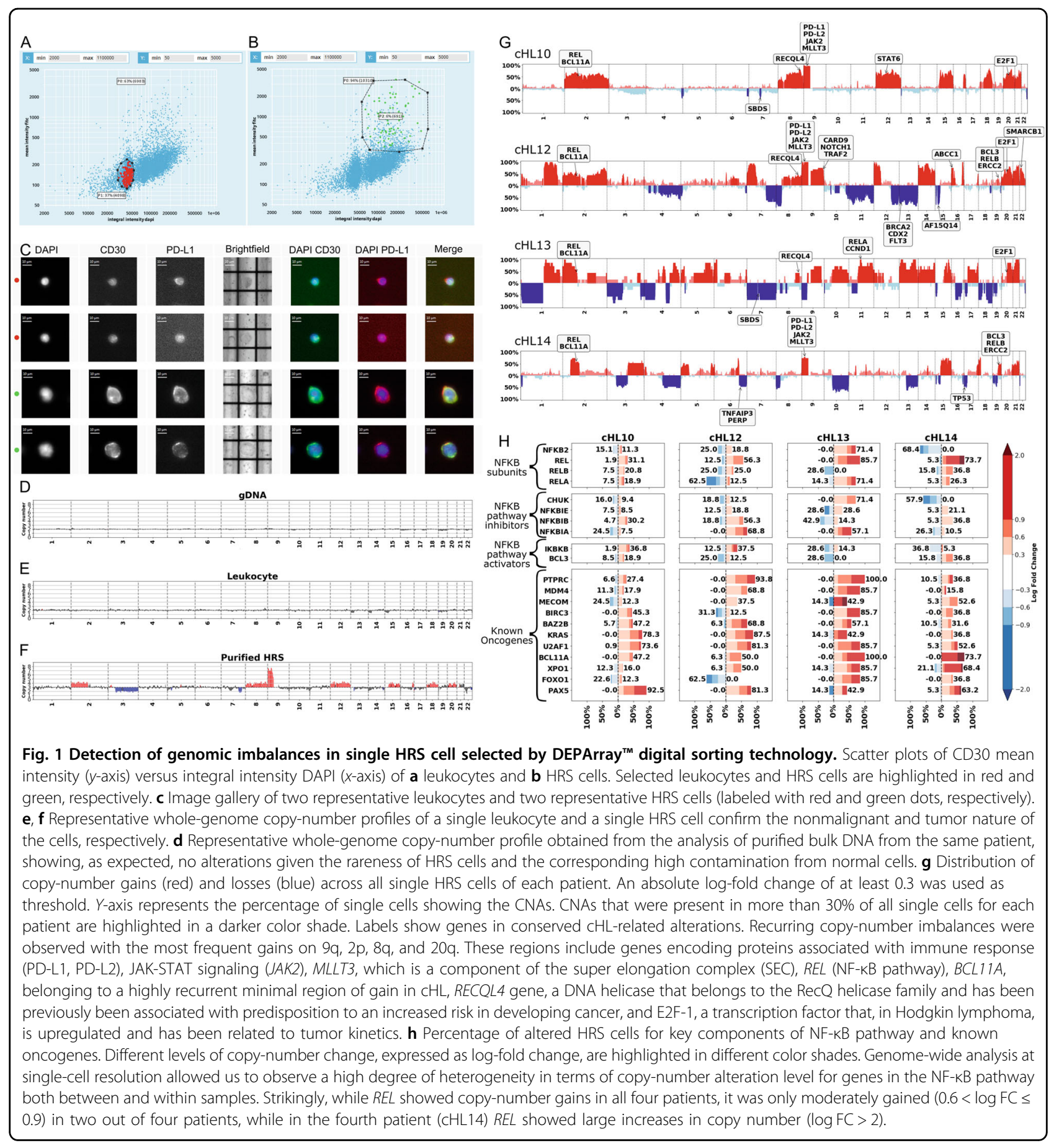

signal intensity and cell/nucleus morphology were employed, in combination with CD30 signal intensity, to discriminate between small and rounded diploid leukocyte cells, with no or basal CD30 expression, and larger hyperdiploid HRS cells showing higher CD30 expression (Fig. 1a-c).

Furthermore, as PD-L1 is often overexpressed in HRS cells, its staining signal intensity was analyzed for all selected HRS cells and compared with the respective leukocyte population showing a much higher signal intensity in HRS cells than in leukocytes, in each analyzed patient (Supplementary Fig. 5).

A total of 213 CD30+ cell recoveries, $27.7 \%$ of which had rosetting $\mathrm{T}$ cells (Supplementary Fig. 6), and 87 CD30 - cell recoveries were performed from tissue samples collected from four cHL patients (Supplementary 
Table 1). Following filtering (Supplementary Methods and Supplementary Figs. 7-9), genome-wide copy-number profiles of $148 \mathrm{CD} 30+$ cells and of 87 CD30- putative leukocytes have been successfully obtained by low-pass whole-genome sequencing. Library success rate was highly related to FFPE tissue quality (Supplementary Fig. 10). Most CD30+ cells (98.0\%) showed genomic alterations, while all CD30- cells showed a flat profile, confirming that the selection employed was effective in isolating aberrant cells (Fig. 1e, f). Figure $1 d$ shows a representative whole-genome copy-number profile obtained from analysis of purified bulk DNA from the same patient, showing, as expected, no alterations given the rareness of HRS cells and high contamination from normal cells.

Out of 148 CD30+ single cells, $79.7 \%$ had large CNA calls ( $>20 \mathrm{Mbp}$; $P$ value $<0.01$ ), and on average, 36.2 segments (11.0 copy-number loss segments; 25.2 copynumber gain segments) were identified per sample accounting for $27.0 \%$ of the genome with values ranging from $1 \%$ up to $57.5 \%$ of genome. Altered segment lengths ranged from $78 \mathrm{kbp}$ to $243 \mathrm{Mbp}$ (average $=32.5 \mathrm{Mbp}$ ) for gains and from 1 to $200 \mathrm{Mbp}$ (average $=27.9 \mathrm{Mbp}$ ) for losses. Among altered regions, a preponderance of gains was observed, contributing, on average, to $70 \%$ of aberrant genome.

Figure 1g shows the composite profiles summarizing the imbalances affecting the autosomes across all single cells analyzed for each patient. Some of the detected CNAs were conserved across patients and patient's single cells, while others were present only on a subset of samples. Among conserved ones, CNAs were detected in regions known to harbor recurrent gains (chromosomes 2p, 8q, and $9 \mathrm{q}$ ) and losses in $\mathrm{cHL}^{8}$. These regions contain genes of pathways known to be altered in cHL, like REL/NF-kB (nuclear factor-kB) and JAK/STAT pathways, which may be involved in the constitutive activation of proliferative and antiapoptotic phenotype of HRS cells 9 . The constitutively activated NF- $\kappa B$ pathway in HRS has been extensively reported and has been related to copy-number gains of genomic regions containing positive regulators like $R E L$ and MAP3K14 $4^{9,10}$.

One patient displayed a high-level amplification of $R E L$ gene together with the copy-number loss of NF- $\mathrm{kB}$ inhibitor $C_{H U K}^{9}$ (Fig. 1h). CNAs in regions adjacent to REL comprised the FOXO1 gene (RNA translocation) and regulatory proteins, including $\mathrm{p} 53$, which has been reported to be deregulated in cHL. In the same region, we detected alterations ranging from few copy gains to amplification of $B C L 11 A^{11}$, which has been shown to be involved in lymphomas pathogenesis 9 .

High levels of genetic imbalances were detected in several oncogenes, already reported as altered in CHL (MDM4 and $U 2 A F 1$ ), and in other lymphoproliferative malignancies.
Finally, the region containing $P D-L 1 / P D-L 2 / J A K 2$ showed gains in most malignant cells of three patients. Interestingly, these alterations displayed highly variable copy-number levels between different HRS cells of the same patient, ranging from polysomy to amplifications, in agreement with a previous report showing a high heterogeneity of $P D-L 1$ copy-number levels, across different cells in the same patient ${ }^{3,4,12}$ (Fig. 2a, b). Noteworthy, one patient displayed, in almost all analyzed HRS, a copynumber loss of PD-L region, which has not been reported before. However, the same patient presented a highly altered genomic profile, with whole-chromosome gains and losses, and the loss of PD-L region may be a consequence of a massive chromosomal instability. Recently, the use of PD-1 blockade has been demonstrated as an effective therapeutic regimen in cHL patients with relapsed/refractory disease ${ }^{12,13}$, and PD-1 blockade effectiveness has been correlated to PD-L locus amplification detected using fluorescence in situ hybridiza$\operatorname{tion}^{3,12}$, a low-throughput and labor-intensive technology, with a limited number of probes multiplexed in a single experiment. Next-generation sequencing-based absolute copy-number profiling of single HRS may provide a valuable tool to uncover alterations at genome-wide level that can be useful for predicting outcome of a specific treatment regimen, for example, for the adoption of immune therapy.

The ability to characterize copy-number profiles at single-cell resolution allowed to zoom in on single patients revealing subclonal copy-number events. In particular, patient cHL10, for which 106 HRS single cells were individually characterized, showed copy-number loss regions in chromosomes 3,6 , or 7 , constituting about $13 \%, 5 \%$, and $7 \%$ of the total of patient cells analyzed, respectively (Fig. 2a, black boxes). Noteworthy, most clonal alterations detected were gains in highly recurrent genomic locations containing driver genes such as $P D-L 1 / P D-L 2$ and $R E L$, while subclonal alterations in examined cases were mainly losses in regions containing tumor suppressors (TNFAIP3 and CDKN1A) and genes associated with DNA repair $(A T R)$. ATR has already been proposed to have a role in lymphomagenesis in HL as deletions and insertions in the gene have been shown to cause a delay/abrogation in double-strand break and single-strand break repair and defects in the accumulation of $\mathrm{p} 53^{14}$. Late mutations in cancer genes involved in maintenance of genome integrity through DNA damage response and repair have already been observed also in other cancer types, and it has been proposed that such mutations may provide advantages to emerging subclones later in evolution ${ }^{15}$.

Various techniques have been employed to overcome the hurdle of assessing HRS cells' genetic alteration in cHL patients due to the rareness of target tumor cells. 


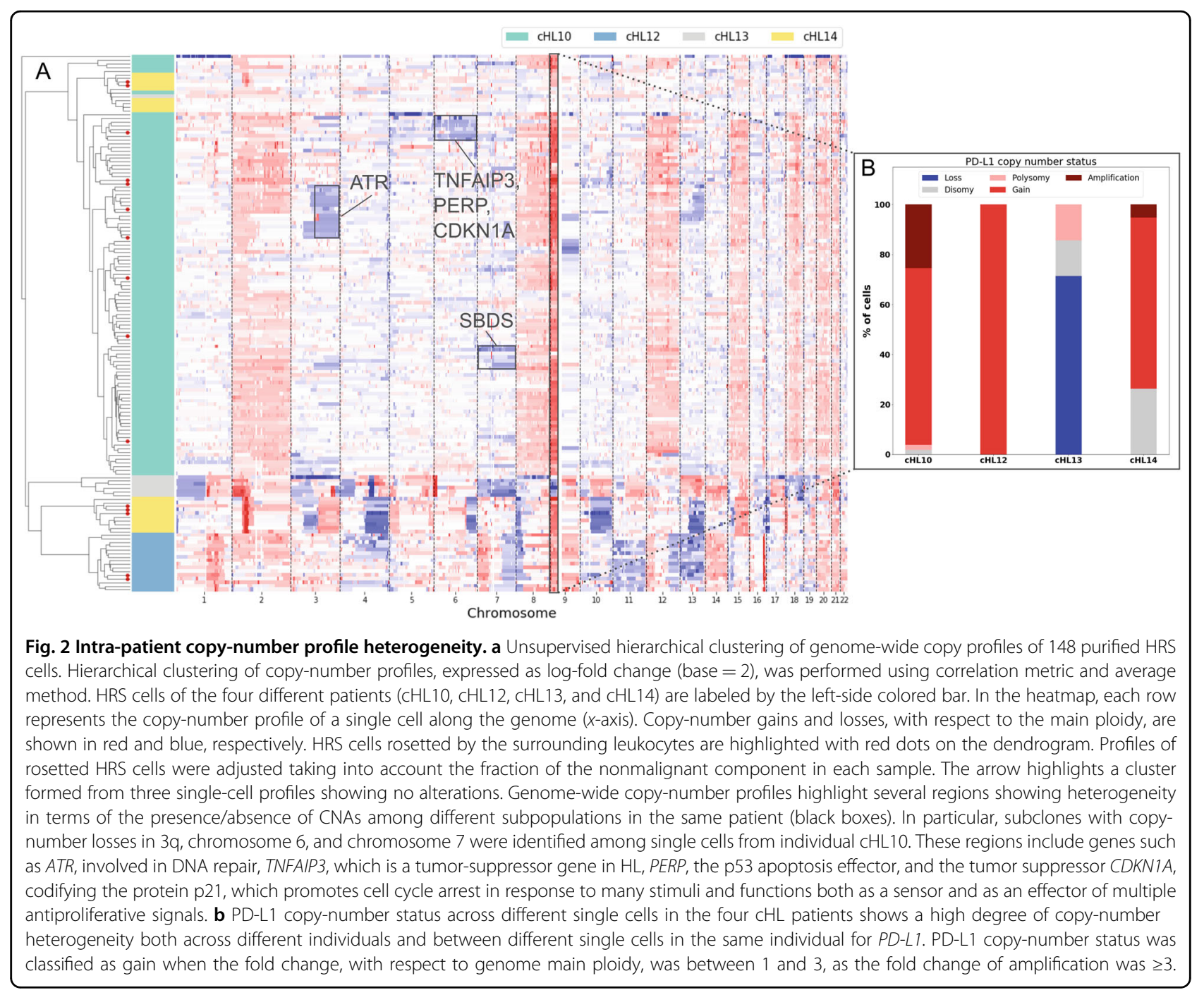

However, the approaches proposed up to now only achieved a partial enrichment of tumor component and did not allow to disentangle subclonal heterogeneity, which may be important for tumor evolution and development, as recently suggested by studies in other tumor types ${ }^{15}$. The workflow presented in this paper addresses the challenges of genetic characterization caused by low tumor cellularity, allowing, for the first time in $\mathrm{cHL}$, to perform genome-wide copy-number profiling at the single-cell level.

Finally, we feel that it will be important in the future to perform additional studies to assess the extent of heterogeneity across larger patient cohorts and to identify potentially clinically significant biomarkers for tumor evolution and response to therapeutic regimens.

\section{Conflict of interest}

C.M., A.F., C.F., M.G., C.B., P.T., R.L., A.R., C.B., G.B., G.M., F.F., and N.M. are employees of Menarini Silicon Biosystems S.p.A.

\section{Publisher's note}

Springer Nature remains neutral with regard to jurisdictional claims in published maps and institutional affiliations.

Supplementary Information accompanies this paper at (https://doi.org/ 10.1038/s41408-019-0256-y).

Received: 23 August 2019 Revised: 5 November 2019 Accepted: 7 November 2019

Published online: 21 November 2019

\section{References}

1. Marafioti, T. et al. Hodgkin and Reed-Sternberg cells represent an expansion of a single clone originating from a germinal center B-cell with functional immunoglobulin gene rearrangements but defective immunoglobulin transcription. Blood 95, 1443-1451 (2000).

2. Vardhana, S. \& Younes, A. The immune microenvironment in Hodgkin lymphoma: T cells, B cells, and immune checkpoints. Haematologica 101, 794-802 (2016).

3. Roemer, M. G. M. M. et al. Major histocompatibility complex class II and programmed death ligand 1 expression predict outcome after programmed death 1 blockade in classic Hodgkin lymphoma. J. Clin. Oncol. 36, 942-950 (2018). 
4. Ramchandren, R. et al. Nivolumab for newly diagnosed advanced-stage classic Hodgkin lymphoma: safety and efficacy in the phase II CheckMate 205 Study. J. Clin. Oncol. 37, 1997-2007 (2019)

5. Polzer, B. et al. Molecular profiling of single circulating tumor cells with diagnostic intention. EMBO Mol. Med. 6, 1371-1386 (2014).

6. Carter, L. et al. Molecular analysis of circulating tumor cells identifies distinct copy-number profiles in patients with chemosensitive and chemorefractory small-cell lung cancer. Nat. Med. 23, 114-119 (2017).

7. Bolognesi, C. et al. Digital sorting of pure cell populations enables unambiguous genetic analysis of heterogeneous formalin-fixed paraffin-embedded tumors by next generation sequencing. Sci. Rep. 6, 20944 (2016).

8. Hartmann, S. et al. Detection of genomic imbalances in microdissected Hodgkin and Reed-Sternberg cells of classical Hodgkin's lymphoma by arraybased comparative genomic hybridization. Haematologica 93, 1318-1326 (2008).
9. Weniger, M. A. \& Küppers, R. NF-kB deregulation in Hodgkin lymphoma. Semin. Cancer Biol. 39, 32-39 (2016).

10. Borchmann, S. \& Engert, A. The genetics of Hodgkin lymphoma: an overview and clinical implications. Curr. Opin. Oncol. 29, 307-314 (2017).

11. Marti, J. I. et al. Recurrent involvement of the REL and BCL11A loci in classical Hodgkin lymphoma Brief report Recurrent involvement of the REL and BCL11A loci in classical Hodgkin lymphoma. Blood 99, 1474-1477 (2013).

12. Roemer, M. G. M. et al. PD-L1 and PD-L2 genetic alterations define classical hodgkin lymphoma and predict outcome. J. Clin. Oncol. 34, 2690-2697 (2016).

13. Pianko, M. J., Moskowitz, A. J. \& Lesokhin, A. M. Immunotherapy of lymphoma and myeloma: facts and hopes. Clin. Cancer Res. 24, 1002-1010 (2018).

14. Liu, A. et al. ATR alterations in Hodgkin's lymphoma. Oncol. Rep. 19, 999-1005 (2008).

15. Jamal-Hanjani, M. et al. Tracking the evolution of non-small-cell lung cancer. $N$. Engl. J. Med. 376, 2109-2121 (2017). 